\title{
Fast Antenna Far Field Characterization via Sparse Spherical Harmonic Expansion
}

\author{
Benjamin Fuchs, Senior Member, IEEE, Laurent Le Coq, Sébastien Rondineau, Member, IEEE, and Marco \\ Donald Migliore, Senior Member, IEEE
}

\begin{abstract}
A procedure is proposed to significantly reduce the amount of time to characterize three dimensional antenna far field patterns. The measured far field is expanded into spherical harmonics and a sparse recovery algorithm is used to recover the spherical wave coefficients giving access to the field radiated by the antenna everywhere. A small number of measurement points are required since the relevant information of most antenna patterns is concentrated in only a few spherical wave coefficients. Sampling strategies enabling fast spherical scans are discussed which makes the approach both efficient and easy to implement in existing far field measurement facilities. Simulations are first provided to show the potentialities of this compressive sensing based approach. The proposed strategy is then applied to characterize three dimensional far field patterns radiated by several antennas operating in different frequency bands measured in far field in direct line of sight configuration and in a compact antenna test range. Experimental results show that a saving in the number of measurement points up to $70 \%$ can be achieved compared to standard approaches. These results pave the way to a more efficient use of far field measurement facilities.
\end{abstract}

Keywords: Antenna measurements, compact antenna test range, compressive sensing, antenna pattern, spherical harmonics.

\section{INTRODUCTION}

The development of antenna design and manufacturing capabilities enables to produce complex radiating structures that have to fulfill always more challenging technical and cost constraints. In this context, efficient procedures must be proposed to characterize the radiation of antennas exhibiting multibeams and multi-frequencies features and three dimensional (3D) radiation characterization is becoming a standard. Near field characterization techniques are a solution to most of these requirements. However, their practical implementation can be difficult and costly to achieve the demanding positioning tolerances especially as the frequency goes up.

Besides, one of the main drawbacks of far field measurement methods is the large number of data required, and consequently the important measurement time required to collect these data. However, the number of information usually collected in spherical scanning is redundant in many practical cases. Symmetries and smoothness in current distributions reduce the set of the fields that can be radiated by the antenna and hence

Manuscript received xx, 2017; revised xx, xx.

B. Fuchs and L. Le Coq are with the IETR / University of Rennes I, France. (email: \{benjamin.fuchs;laurent.le-coq\} @univ-rennes1.fr). S. Rondineau is with the University of Brasilia, Brazil (email: sebastien@unb.br). M.D Migliore is with the DIEI, University of Cassino e Lazio Meridionale, Italy. (email: mdmiglio@unicas.it). the number of the measurements required to identify this field. In practice, exploiting detailed information on the antenna can reduce the number of measurements. Unfortunately, such strategy requires a field representation using bases that match the antenna, as well as some a-priori information on the antenna, making their practical use cumbersome.

In this paper, a procedure to speed up the antenna 3D characterization in the far field is proposed. It uses a general-purpose basis (spherical harmonics) without explicit a-priori information on the antenna (apart from its maximum dimension and position). The approach is directly applicable to existing far field facilities including compact antenna test ranges without any hardware modifications or limitation on the antenna under test. Inspired by the recent advances in compressive sensing, the main idea is to take advantage of the sparsity of the electromagnetic field representation in the spherical harmonics domain. Indeed, a number of information (for example information on potential symmetries) are implicitly included in the spherical harmonics representation in terms of low value of coefficients. The method takes advantage of this a priori sparseness in the spherical harmonic representation to decrease the number of required measurement points.

Over the last decade, the developments in the field of compressive sensing have significantly changed many aspects in data sampling and processing [1]-[3]. The idea to exploit the sparsity to achieve undersampling rates has found a great relevance in many applications in signal processing, information theory and electrical engineering to name just a few. Despite its great potential, the application of compressive sensing in electromagnetics is relatively new, as reviewed in [4]. Several contributions have recently been made in antenna measurements and diagnostics [5]-[7] but most approaches proposed so far rely on a strong a priori knowledge about the antenna under test, such as the failure-free pattern or a numerical model of the radiating structure.

The applicability of compressive sensing depends on the determination of a proper basis in which the signal, in our case of interest the electromagnetic field, has a sparse representation. The electromagnetic field can be expanded into spherical harmonics [8], [9] and it turns out that the spherical wave spectrum of antennas exhibits sparse properties as noticed by [10] and [11]. Thus, an interesting compressive sensing approach has been applied to spherical near field to far field transformation in [10] and very promising simulation results are shown. A similar approach is used in [11] to speed up spherical near field measurements. The optimization of the coordinate system to further enforce the sparsity of the spher- 
ical wave coefficients is also addressed and a methodology to estimate the phase center position is derived.

In parallel and complementarily to the foregoing research, our approach is dedicated to antenna 3D far field pattern characterization. The measured electrical far field is expanded into a spherical harmonic basis leading to a sparse representation of the field. Efficient and readily available algorithms are then used to reconstruct the 3D field from a reduced number of measurements compared to standard approaches as described in Section II. A special focus is given in the sampling strategy enabling a fast and easy to implement spherical scan. Numerical simulations are provided in Section III to show that compressive sensing approaches work also well with spherical harmonics matrices. Experimental results, obtained in far field measurement facilities (both line of sight and compact antenna test range configuration), of several radiating structures operating in various frequency bands are shown in Section IV to confirm the interest of the proposed procedure.

\section{Problem Formulation}

\section{A. Spherical Harmonic Expansion}

The electric far field (co- and cross- polarization) can be expanded into scalar spherical harmonics as follows:

$$
E(\theta, \varphi)=\sum_{l=0}^{\infty} \sum_{m=-l}^{l} c_{l m} Y_{l}^{m}(\theta, \varphi)
$$

where $c_{l m}$ are the spherical wave coefficients of degree $l$ and order $m$ [8], [12]. The scalar spherical harmonics are:

$$
Y_{l}^{m}(\theta, \varphi)=\sqrt{\frac{(2 l+1)(l-m) !}{4 \pi(l+m) !}} P_{l}^{m}(\cos \theta) e^{i m \varphi}
$$

where $P_{l}^{m}(\cos \theta)$ are the associated Legendre polynomials of degree $l$ and order $m$.

The knowledge of the spherical wave coefficients $c_{l m}$ enables to compute the antenna far field and also the field radiated everywhere except in the smallest sphere enclosing the radiating structure. The total power radiated by the antenna and therefore its directivity $D$ can easily be derived:

$$
D(\theta, \varphi)=\frac{E(\theta, \varphi)}{\sum_{m, l}\left|c_{l m}\right|^{2} /(4 \pi)} .
$$

When numerically implemented, the series in (1) require a truncation order $L$ and the angles $(\theta, \varphi)$ are discretized into $M$ directions. As detailed in [12], the far field is well approximated when the truncation order $L$ is equal to $\left[\frac{2 \pi}{\lambda} a\right]+n_{1}$ where $[x]$ stands for the integer part of $x, \lambda$ is the free space wavelength, $a$ the radius of the smallest sphere enclosing the antenna and the positive integer $n_{1}$ is commonly set to 10 .

The electric far field, i.e. the equation (1), can be written in a matrix form:

$$
\mathbf{y}=\mathbf{A x}
$$

where $y_{m}=E\left(\theta_{m}, \varphi_{m}\right)$ are the measurement points and the vector $\mathbf{x} \in \mathbb{C}^{N}$ gathers the spherical wave coefficients $c_{l m}$ to be determined. The columns of the (sensing) matrix $\mathbf{A} \in$ $\mathbb{C}^{M \times N}$ are the $N$ scalar spherical harmonics $Y_{l}^{m}$ (2) with $N=(L+1)^{2}$.

\section{B. Computation of the Spherical Wave Coefficients}

The determination of the spherical wave coefficients $c_{l m}$ from the measured radiated far field $E(\theta, \varphi)$ can be done analytically from the tangential components of the electric field thanks to the orthogonality properties of the spherical harmonics [9]. A popular numerical approach, based on discrete Fourier Transforms, has been proposed by Hansen [12]. It is both computationally efficient and easy to implement because $\Delta \theta=\Delta \varphi$ but it requires a high number of measurements points since $M=2(2 L+1)(L+1)$. Other numerical methods have been applied to solve the linear inverse problem (4), i.e. to find $\mathbf{x}$ from $\mathbf{y}$. Because of the bad conditioning of $\mathbf{A}$, regularization procedures such as Tikhonov regularizer or the truncated singular value decomposition [13] are necessary to stabilize the inversion. These strategies work fairly well as long as the number of measurements $M$ is greater than the number of unknowns $N$. When $M$ is (slightly) lower than $N$, the success of these approaches depends highly on the sampling of the field, as discussed in the next Section.

The idea to use a sparse prior to recover spherical wave coefficients has been first, to our best knowledge, recently proposed by [10], [11] and applied successfully on antenna spherical near field patterns. Many antenna patterns can indeed be accurately described by using a few spherical coefficients $K$, where $K \ll N$, which means that the spherical wave magnitude spectrum is often sparse. This assumption is all the more true when the antenna is well positioned in its phase center (as discussed in [11]) and / or exhibits some geometrical symmetry.

One convenient way to foster sparse spherical wave coefficients is to use the $\ell_{1}$-norm $\left(\|\mathbf{x}\|_{1}=\sum_{i}\left|x_{i}\right|\right)$ as a regularizer [1], [2]. The inverse problem to solve reads:

$$
\min _{\mathbf{x}}\|\mathbf{x}\|_{1} \text { subject to }\|\mathbf{y}-\mathbf{A} \mathbf{x}\|_{2} \leq \varepsilon
$$

where the positive parameter $\varepsilon$ enables to handle the inevitable misfit errors due to measurement noise and uncertainties. The convex optimization problem (5) can be solved efficiently by many generic optimization routines, such as [14]-[16]. We have used SPGL1 [14], a MatLab solver for large-scale onenorm regularized least squares in this paper.

\section{Discussion on the Field Sampling}

The sensing matrix A plays a crucial role in the determination of the sparse spherical wave coefficients $\mathbf{x}$. In short, the mutual coherence, null space property or restricted isometry property of $\mathbf{A}$ are metrics (sometimes very hard to evaluate) that enable to assess the recoverability of $\mathrm{x}$ solving (5). We refer interested readers to [17] for a complete overview on the properties of $\mathbf{A}$ and the associated sparse recovery performances.

Loosely speaking, sparse recovery uses random projections to estimate the sparse unknown from a set of data. This requires to randomly select a (hopefully low) number of measurement positions on the observation surface. However, a completely random selection is generally not the best option [18].

For antenna far field pattern measurements, the only degree of freedom to design $\mathbf{A}$ is the choice of the sampling points 
$\left(\theta_{m}, \varphi_{m}\right)$. Ideally, each measurement point $y_{m}$ should bring an additional (non redundant) information. Thus, sampling the field with an constant angular step $\Delta \theta$ and $\Delta \varphi$ brings an oversampling and consequently an important redundancy near the poles. It is therefore not the best choice to build A. Low redundant sampling grids have been the object of research using both theoretical and heuristic approaches. A simple sampling strategy consists in sampling the sphere while keeping a constant solid angle for all $\theta$ angle, which yields:

$$
\Delta \varphi=\frac{\Delta \theta}{\sin \theta}
$$

where $\Delta \theta$ is constant. This sampling strategy is from now on called 'igloo' sampling. It is easy to implement in practice and enables fast spherical scans, since for each latitude $\theta_{i}$ the azimuthal step $\Delta \varphi_{i}$ is constant.

Starting from a rigorous theoretical approach, Bucci et al. proposed a 'minimum redundant' sampling strategy [19]. This method is based on the evaluation of the spatial bandwidth of the 'reduced field', that is a smoother version of the field on the observation surface obtained substracting a proper phase function and introducing a proper parameterization on the coordinate curves. The knowledge of the bandwidth allows to sample the field at the Nyquist rate, obtaining the following positions of the sampling points in spherical coordinates $(\theta, \varphi)$ [19]:

$$
\begin{aligned}
\Delta \theta & =\frac{2 \pi}{2[\chi \beta a]+1} \\
\Delta \varphi & =\frac{2 \pi}{2\left[\chi_{\theta} \beta a \sin (\theta)\right]+1} \\
\chi_{\theta} & =1+(\chi-1) \sin (\theta)^{-2 / 3}
\end{aligned}
$$

where $\beta=2 \pi / \lambda$ is the free space wavenumber, $a$ is the radius of the smallest sphere enclosing the antenna and $\chi$ is an oversampling factor that controls the truncation error in the sampling series. In practical cases, $\chi$ is slightly larger than 1 . It is interesting to note that the 'igloo' and Bucci's sampling lead, for similar values of $\Delta \theta$, to very close sampling positions. The main difference comes from the slight oversampling near the poles of the spherical reference system brought by Bucci's theory.

\section{Numerical Results on Sparse ReCOVERY PERFORMANCES}

Theoretical results on sparse recovery performances have been first established for Gaussian matrices A [1]. More recently, investigations have been carried out for spherical harmonic matrices [20]. The idea is to understand how sparse spherical wave coefficients can be recovered from a small number of randomly chosen samples on the sphere using $\ell_{1}$ minimization, i.e. solving (5).

We perform here numerical simulations in the framework of antenna far field pattern characterization. Let us consider random spherical wave coefficients $\mathrm{x}$ of size $N$ with a given sparsity level $K / N$. These coefficients are normalized such that the total power radiated by the antenna remains constant for all cases. A number $M$ of sampling points are chosen randomly from a dense 'igloo' sampling on the sphere and the basis pursuit problem, i.e. the $\ell_{1}$ minimization problem (5) with $\epsilon=0$ is solved. The solution is denoted $\tilde{\mathbf{x}}$. The sparse reconstruction is assumed to be successful when the Mean Square Error (MSE):

$$
\mathrm{MSE}=20 \log _{10}\left(\frac{\mathbf{x}-\tilde{\mathbf{x}}}{\sqrt{N}}\right)
$$

is lower than $-40 \mathrm{~dB}$.

We plot for various sampling ratio $M / N$ and two sparsity levels $K / N$ of 0.25 and 0.5 , the probability of having a successful reconstruction over 100 trials in Fig. 1. The total number of spherical wave coefficients $N$ is set to 400. An

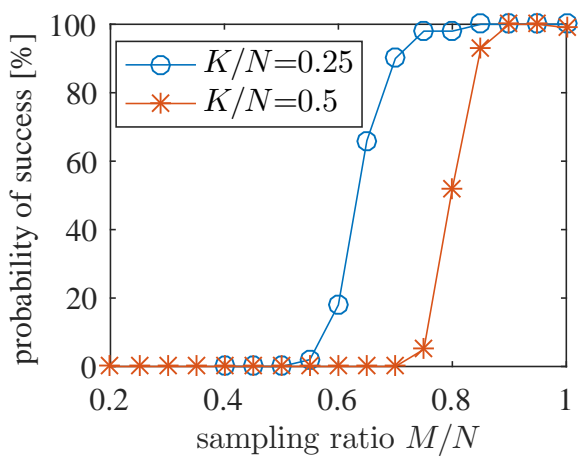

Fig. 1: Probability of success of the sparse reconstruction in the case $N=400$ for various sampling ratio $M / N$ and sparsity levels $K / N$.

undersampling of about $20 \%$ can be applied to perfectly reconstruct a spherical wave coefficients spectrum having a sparsity level of 0.25 ( 1 coefficient out of 4 is non zero). When half of the coefficients are non zero, the required number of measurement can be reduced of about $10 \%$ using compressive sensing techniques.

The phase transition diagram, introduced by Donoho and Tanner in compressive sensing [21], shows the probability of perfect reconstruction as a function of the sampling ratio $\delta=M / N$ (also called level of undetermindness) and the sparsity level here $\rho=K / M$. A perfect reconstruction from undersampled measurements $\delta$ is achieved until the sparsity level $\rho$ remains below a threshold value. Although the sparsity level of the spherical wave coefficients of an antenna is not a priori known, the phase transition diagram enables to estimate the range of measurement undersampling that can be expected. The phase transition diagram is plotted in Fig. 2 and we notice a sharp transition between a perfect recovery (in red) and a bad one (in blue), this behavior is typical in compressive sensing. When $M / N=1$, it means that the number of unknown is equal to the number of measurement points, the use of the 'igloo' sampling strategy ensures then a good reconstruction for all sparsity levels.

Note that this study has already been carried out in [10] with different settings which explains that slightly different results are obtained. 


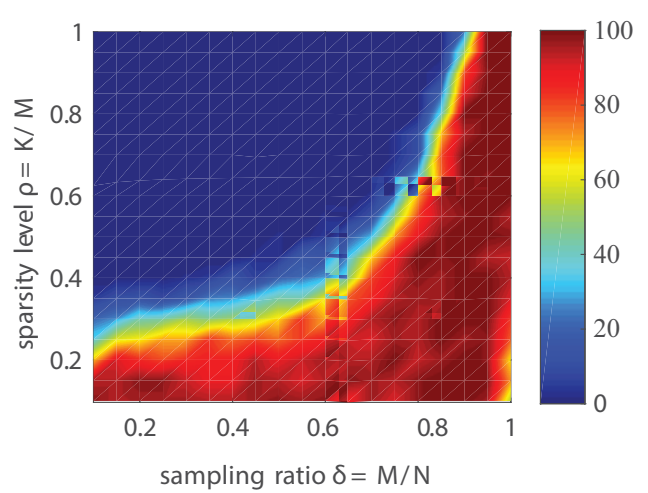

Fig. 2: Phase transition diagram for $N=400$. The scale indicates the percentage of successful recovery of (sparse) spherical wave coefficients. A perfect recovery / failure is achieved in the red / blue region.

\section{EXPERIMENTAL RESULTS}

Several antennas operating in different frequency bands have been characterized in the far field anechoic chambers (in both line of sight configuration and compact antenna test range) of the Institute of Electronics and Telecommunications of Rennes in France. Their 3D radiated patterns (in the full or half sphere) are used to show the interest of the proposed fast antenna far field characterization approach.

For each antenna, the problem (5) (denoted $\ell_{1}$ ) is solved to determine the $N$ spherical wave coefficients from $M$ measurement points taken on a (coarse) 'igloo' grid. The derived $3 \mathrm{D}$ far field (vector denoted $\mathbf{y}_{\ell_{1}}$ ) is compared to the one obtained applying the Truncated Singular Value Decomposition $\left(\mathbf{y}_{\mathrm{TSVD}}\right)$. The far field measured in a dense sampling points is taken as a reference ( $\mathbf{y}_{\text {Meas. }}$ ).

In order to assess the quality of the proposed method, we compare the patterns $\mathbf{y}_{\ell_{1}}$ and $\mathbf{y}_{\text {TSVD }}$ to the reference one $\mathbf{y}_{\text {Meas. }}$. One way to compare two patterns is to perform a weighted logarithmic difference as proposed in [22]. The idea is to de-emphasize the difference at low pattern levels. Indeed, pattern differences at nulls, for instance, are not meaningful. This weight is computed considering the source of errors that are corrupting the measurements. In an anechoic chamber, the main figure of merit is the reflectivity level $\mathrm{R}$ (ratio between reflected and direct signal). Consequently, one way to build the weight function $w(\mathbf{y})$ is to compute the ratio between the desired signal and the desired signal with a constructive interference (worst case):

$$
w(\mathbf{y})=\frac{|\mathbf{y}|}{|\mathbf{y}|+\mathrm{R}} .
$$

The weight as a function of the measurement level $\mathbf{y}$ for a dynamic of $80 \mathrm{~dB}$ and a reflectivity level of the chamber equal to $-50 \mathrm{~dB}$ is plotted in Fig. 3. The weighted logarithmic difference $\Delta\left(\mathbf{y} / \mathbf{y}_{\text {Meas. }}\right)$ is then:

$\Delta\left(\mathbf{y} / \mathbf{y}_{\text {Meas. }}\right)=w\left(\mathbf{y}_{\text {Meas. }}\right)\left|20 \log _{10}\left(\left|\mathbf{y}_{\text {Meas. }}\right|\right)-20 \log _{10}(|\mathbf{y}|)\right|$.

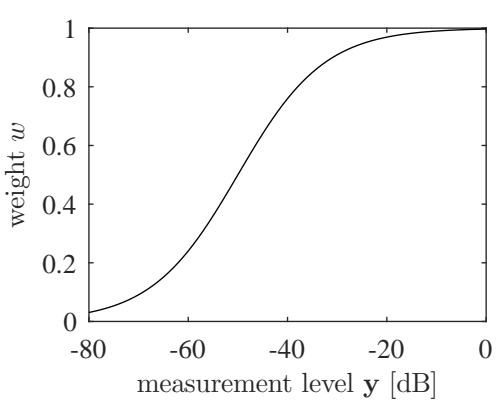

Fig. 3: Weight function to de-emphasize the difference between patterns at low field level values. The reflectivity level $R$ of the chamber is set to $-50 \mathrm{~dB}$.

\section{A. Reflectarray}

A reflectarray composed of 193 cells, designed by Thalès Alenia Space in the framework of the project R3MEMS, has been characterized in X band. A total number of 32761 copolar and cross-polar data have been measured on a far-field halfsphere (step of $0.5^{\circ}$ in $\theta$ and $2^{\circ}$ in $\varphi$ ), these data are our reference denoted $\mathbf{y}_{\text {Meas. }}$, see Fig. 4(b).

We extract, from this dense sampling, the points that as close as possible to the 'igloo' sampling represented in Fig. 4(a). The total number of measurement points is then $M=871$. From these $M$ measurement points, we reconstruct the $N$ complex spherical wave coefficients $\mathbf{x}$ where $N=(55+1)^{2}=3136$ using the proposed $\ell_{1}$ minimization approach (5) and the TSVD approach. We derived from these two solutions $\mathbf{x}$ the far field patterns $\mathbf{y}_{\ell_{1}}$ and $\mathbf{y}_{\text {TSVD }}$.

The patterns and differences with respect to $\mathbf{y}_{\text {Meas. }}$ are plotted in $3 \mathrm{D}$ in Fig. 5 and in $2 \mathrm{D}$ in Fig. 6. The proposed $\ell_{1}$ algorithm leads to a better far field interpolation since $\mathbf{y}_{\ell_{1}}$ is closer to $\mathbf{y}_{\text {Meas. }}$ than $\mathbf{y}_{\mathrm{TSVD}}$, this is clearly visible in the main lobes of Fig. 6(a) and (c).

A significant reduction of the required number of measurement points for a proper interpolation of the 3D far field. In this example, a saving of more than $70 \%$ measurement points is achieved compared to standard approaches that require roughly $M \approx N$.

\section{B. Standard Gain Horn}

A standard gain horn operating in Ka-band has been characterized at $26 \mathrm{GHz}$ in the IETR compact antenna test range. A total number of 5659 copolar and cross-polar data points have been measured on a far-field half-sphere, these data are

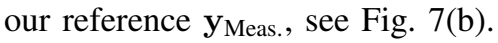

From this dense sampling, the points that are as close as possible to the 'igloo' sampling represented in Fig. 7(a) are considered. The total number of measurement points is then $M=339$. From these $M$ points, we reconstruct the $N$ complex spherical wave coefficients $\mathbf{x}$ where $N=(30+$ $1)^{2}=961$ using the proposed $\ell_{1}$ minimization approach (5) and the TSVD approach. We derived the corresponding far field patterns $\mathbf{y}_{\ell_{1}}$ and $\mathbf{y}_{\text {TSVD. }}$. The patterns and differences with respect to the measurement are plotted in $3 \mathrm{D}$ in Fig. 


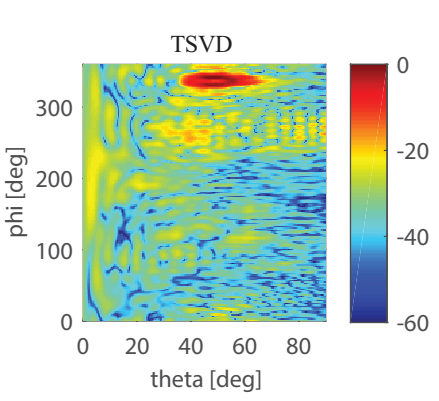

(a)

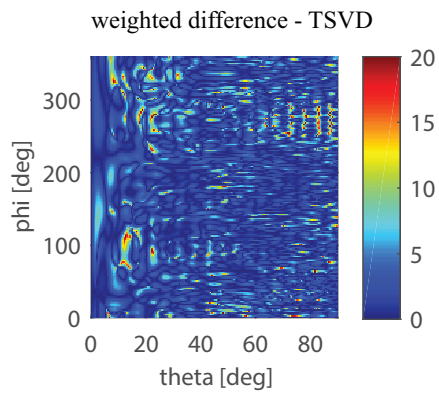

(b)

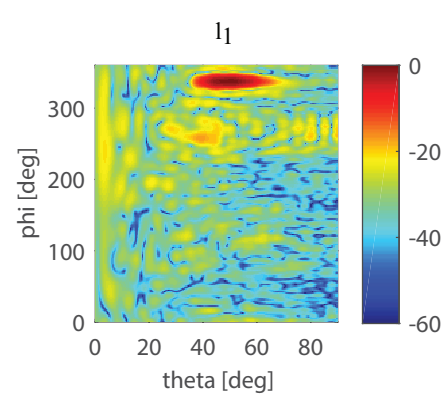

(c)

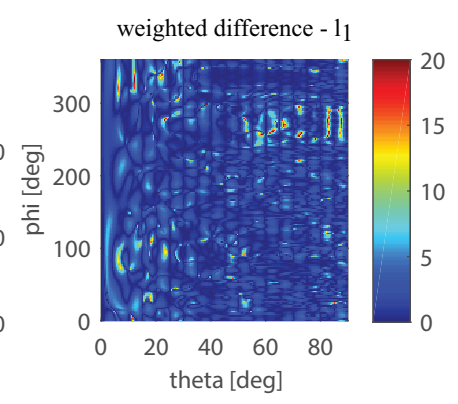

(d)

Fig. 5: 3D far field mappings in $\mathrm{dB}$ radiated by the reflectarray and interpolated from the 'igloo' sampling with $M=871$ points and for a truncation order of $L=55$ ( $N=3136$ spherical wave coefficients). Far field patterns and weighted differences between exact and reconstructed field obtained $(\mathrm{a}, \mathrm{b})$ by the TSVD approach and $(\mathrm{c}, \mathrm{d})$ by the proposed the $\ell_{1}$ minimization procedure (5).

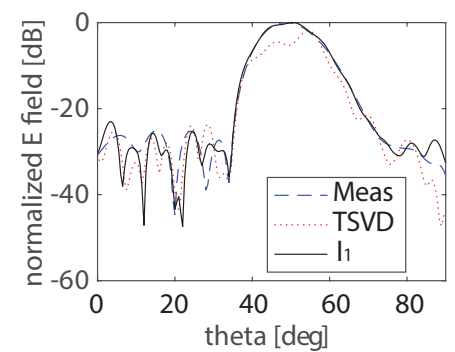

(a)

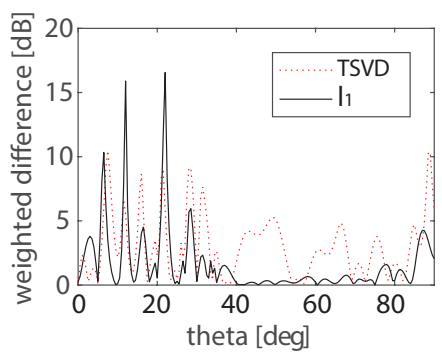

(b)

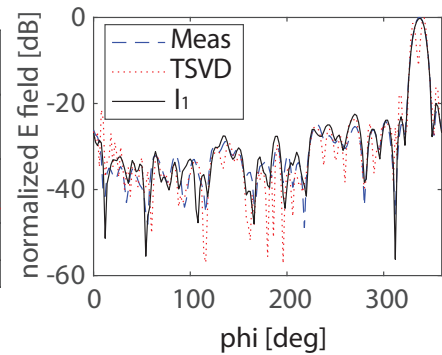

(c)

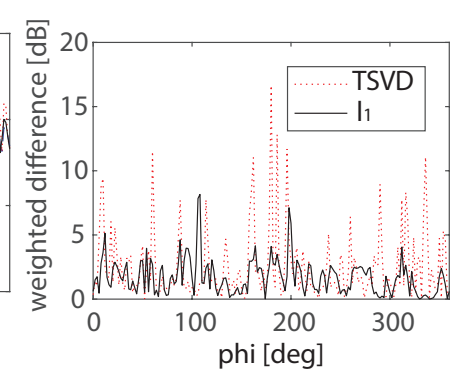

(d)

Fig. 6: Far field patterns in the main cutting planes radiated by the reflectarray and interpolated from the 'igloo' sampling. $(a, c)$ Normalized patterns and $(b, d)$ corresponding weighted differences.

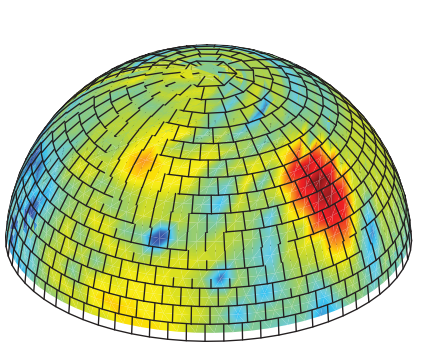

(a)

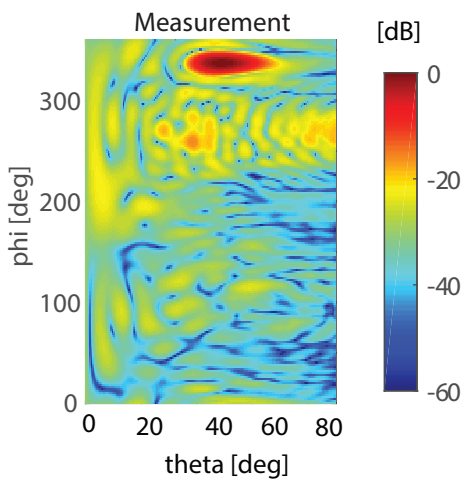

(b)

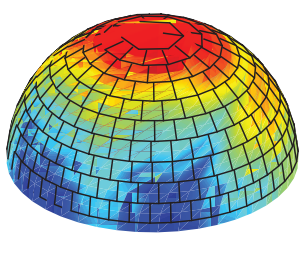

(a)

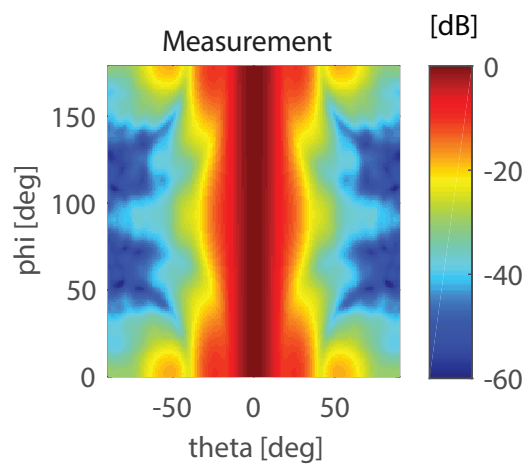

(b)
Fig. 4: Far field patterns radiated by the reflectarray at $12 \mathrm{GHz}$ : (a) sampling using the 'igloo' technique with $\Delta \theta=5^{\circ}$ ( $M=871$ sampling points) and (b) measured 3D field mapping $\mathbf{y}_{\text {Meas. }}$.

8 and in 2D in Fig. 9. The proposed $\ell_{1}$ algorithm better interpolates the far field than the TSVD, as shown in the 2D cutting plane difference Fig. 6(b). We can also notice the good reconstruction of the pattern phase in Fig. 6(c). In this example, compared to standard approaches for which $M \approx N$, less than $1 / 3$ of the points are needed for a good reconstruction of the $3 \mathrm{D}$ pattern.
Fig. 7: Far field patterns radiated by the standard gain horn at $26 \mathrm{GHz}$ : (a) sampling using the 'igloo' technique with $\Delta \theta=$ $8^{\circ}$ ( $M=339$ sampling points) and (b) measured 3D field mapping $\mathbf{y}_{\text {Meas. }}$.

\section{Flat Luneburg Lens}

A flat metallic Luneburg lens antenna has been designed in the frame of MERLIN, a joint laboratory of Thalès Alenia Space and IETR. Details about this radiating structure are available in [23]. A total number of 65341 copolar data have been measured at $12 \mathrm{GHz}$ on a far-field full-sphere which makes possible the computation of the directivity (3). These 


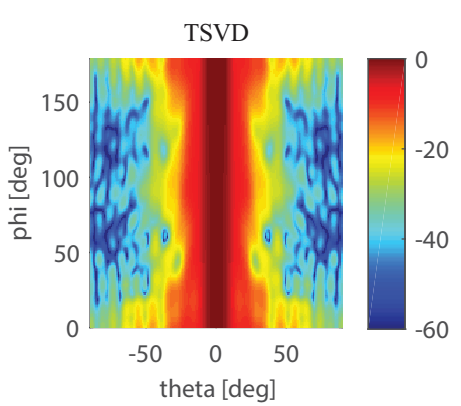

(a)

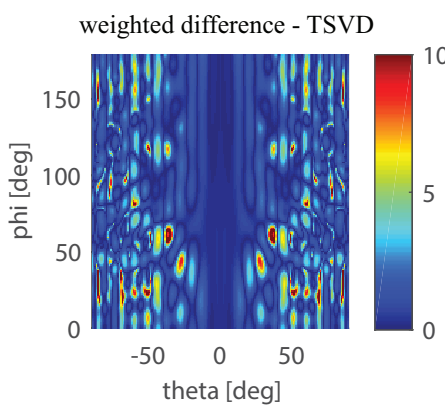

(b)

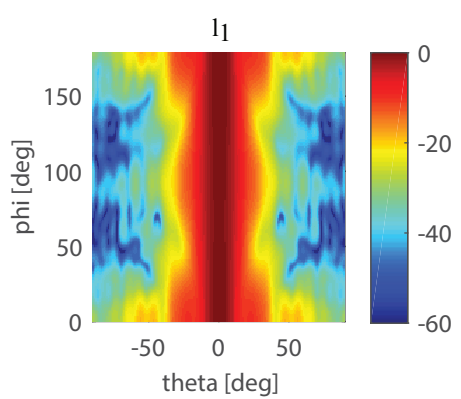

(c)

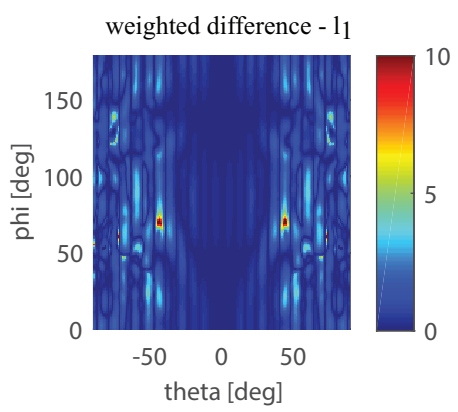

(d)

Fig. 8: 3D far field mappings in $\mathrm{dB}$ radiated by the standard gain horn and interpolated from the 'igloo' sampling with $M=339$ points and for a truncation order of $L=30$ ( $N=961$ spherical wave coefficients). Far field patterns and weighted differences between exact and reconstructed field obtained $(\mathrm{a}, \mathrm{b})$ by the TSVD approach and $(\mathrm{c}, \mathrm{d})$ by the proposed the $\ell_{1}$ minimization procedure (5).

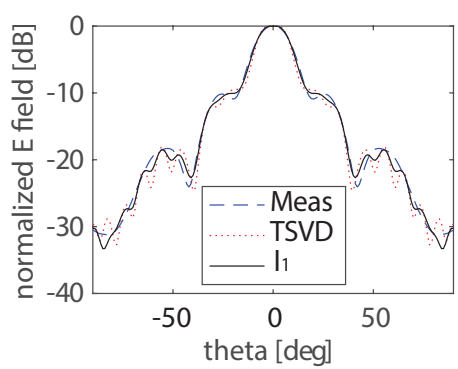

(a)

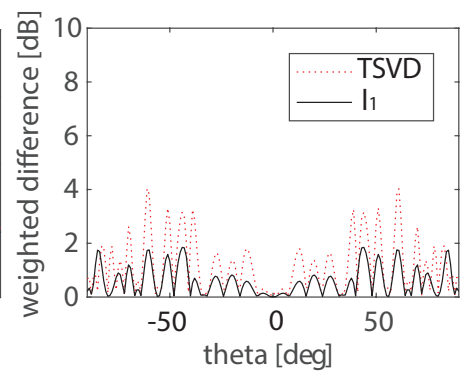

(b)

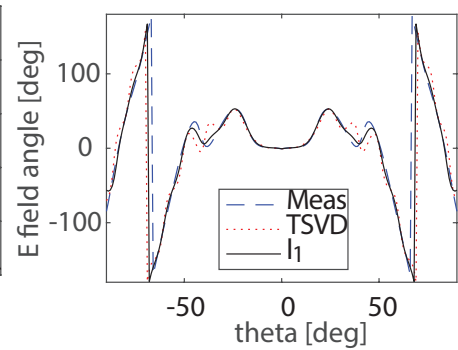

(c)

Fig. 9: Far field patterns in the cutting plane $\varphi=0^{\circ}$ radiated by the standard gain horn and interpolated from the 'igloo' sampling: (a) Normalized amplitude pattern, (b) weighted difference and (c) phase patterns.

data are our reference $\mathbf{y}_{\text {Meas. }}$ and plotted in Fig. 10(b) where we clearly see the lens focusing effect along one plane $\varphi=90^{\circ}$, it is a flat (2D) lens.

The points, that as close as possible to the 'igloo' sampling represented in Fig. 10(a), are considered. We reconstruct from these $M=2057$ points, the $N$ complex spherical wave coefficients $\mathbf{x}$ where $N=(55+1)^{2}=3136$ using the proposed $\ell_{1}$ minimization approach (5) and the TSVD approach. The patterns $\mathbf{y}_{\ell_{1}}$ and $\mathbf{y}_{\text {TSVD }}$ and the differences $\Delta\left(\mathbf{y}_{\ell_{1}} / \mathbf{y}_{\text {Meas. }}\right)$ and $\Delta\left(\mathbf{y}_{\text {TSVD }} / \mathbf{y}_{\text {Meas. }}\right)$ are plotted in $3 \mathrm{D}$ in Fig. 11 and in $2 \mathrm{D}$ in Fig. 12.

The proposed approach leads to a better far field interpolation as it can be easily seen from the 2D cutting plane of Fig. 12(d). This is confirmed when computing the directivity from the spherical waves coefficients: $23.2 \mathrm{~dB}$ are obtained with the TSVD while $21.8 \mathrm{~dB}$ with the proposed approach that is closer to the reference directivity of $21.6 \mathrm{~dB}$. In this example, compared to standard approaches with $M \approx N$, about $2 / 3$ of the points are needed for a good reconstruction of the $3 \mathrm{D}$ pattern.

\section{Summary}

Let us summarize the previous experimental results in the Table I. We denote RA, SGH and LL the ReflectArray, Standard Gain Horn and Luneburg Lens antenna respectively.

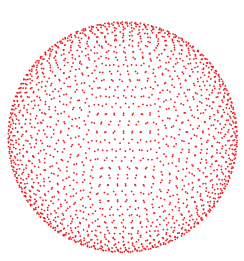

(a)

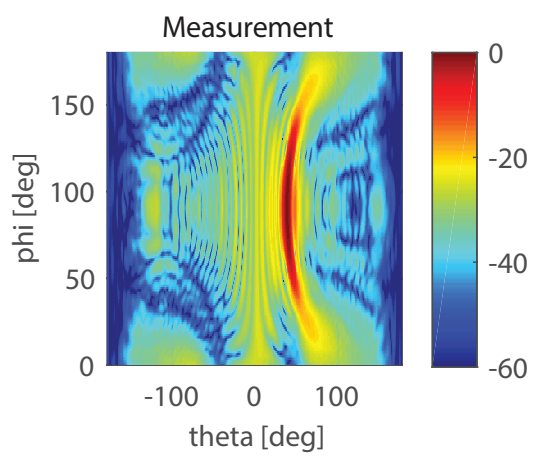

(b)
Fig. 10: (a) Positions of the 'igloo' sampling points, on average $\Delta \theta=4.5^{\circ}$ and $\Delta \varphi=11.2^{\circ}$. (b) Far field pattern radiated by the flat Luneburg lens at $12 \mathrm{GHz}$.

The value $a$ is the radius of the smallest sphere enclosing the antenna and all parts contributing to the radiation, such as the antenna metallic support for instance. Theoretically, the truncation order $L$ of the spherical harmonic series should be chosen greater than $\left[\frac{2 \pi}{\lambda} a\right]$. In practice, the truncation order $L$ is chosen slightly lower than this value and satisfying results are obtained.

The determination of the $N=(L+1)^{2}$ spherical wave 


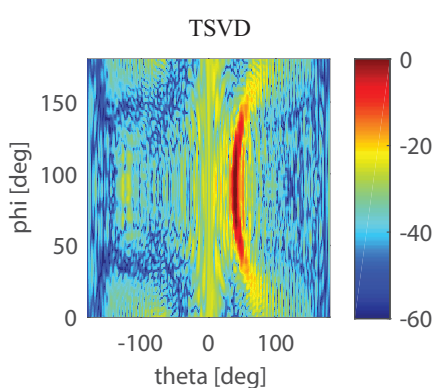

(a)

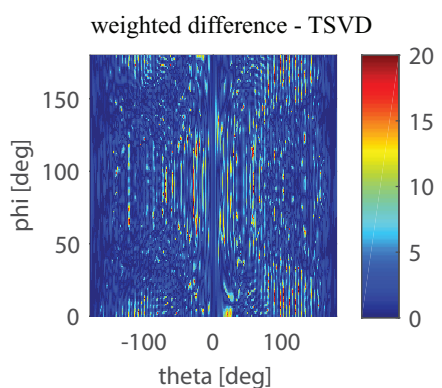

(b)

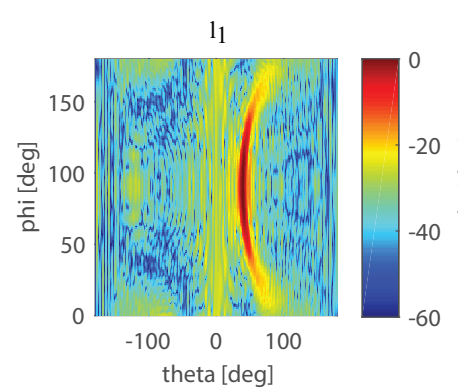

(c)

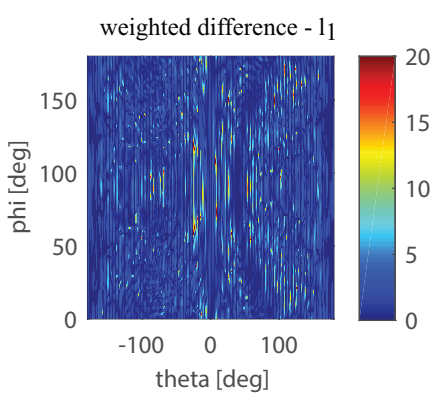

(d)

Fig. 11: 3D far field mappings in $\mathrm{dB}$ radiated by the plate Luneburg lens and interpolated from the 'igloo' sampling represented Fig. 10(a) $M=2057$ points and for a truncation order of $L=55$, i.e. $N=3136$ spherical modes. Far field pattern and weighted difference between exact and reconstructed field obtained $(\mathrm{a}, \mathrm{b})$ by the TSVD approach and $(\mathrm{c}, \mathrm{d})$ by the proposed the $\ell_{1}$ minimization procedure (5).

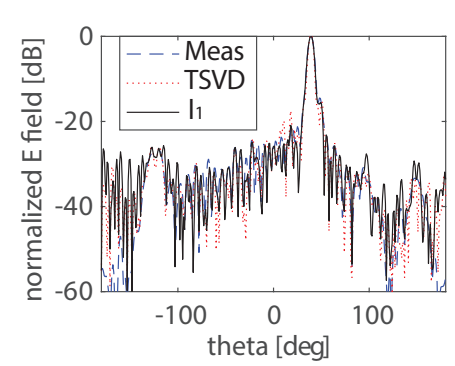

(a)

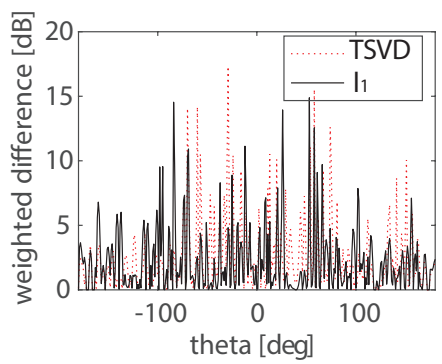

(b)

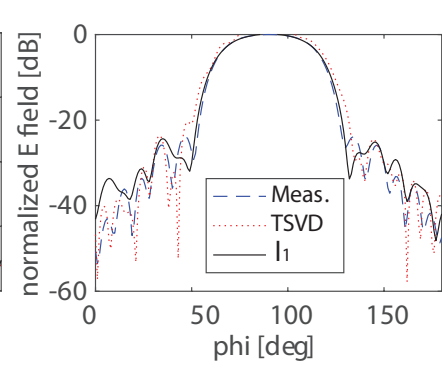

(c)

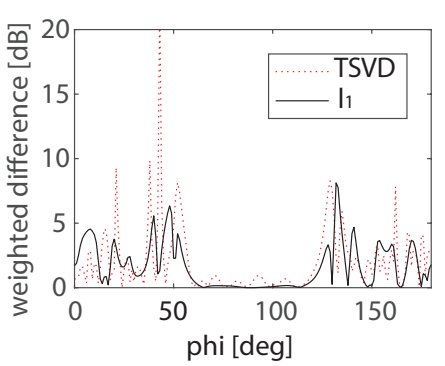

(d)

Fig. 12: Far field patterns in the main cutting planes radiated by the plate Luneburg lens and interpolated from the 'igloo' sampling. $(\mathrm{a}, \mathrm{c})$ Normalized patterns and $(\mathrm{c}, \mathrm{d})$ corresponding weighted differences.

coefficients can be carried out efficiently from a uniform angular sampling $\Delta \theta=\Delta \varphi$ using discrete Fourier Transforms and the number of required measurement points is then $M_{\mathrm{H}}=2(2 L+1)(L+1)$ so approximately $4 N$ (for the full sphere case), the subscript $\mathrm{H}$ stands for Hansen [12].

The far field can be exactly reconstructed from the optimal (in the sense of minimum redundancy) sampling strategy combined with a proper interpolation scheme proposed by Bucci et al. [19], see (7). We denote $M_{\mathrm{B}}$ this number of measurement points.

Finally, the proposed approach reconstructs the $N$ spherical wave coefficients from $M$ measurement points following an 'igloo' sampling via the sparse recovery algorithm (5). Exploiting the sparsity prior on the spherical wave expansion enables us to reduce the number of points, from $M \approx N$ as done with conventional matrix inversion approaches, up to $M=N / 3$. It is important to point out that both Hansen's and Bucci's approaches are exact procedures enabling a perfect reconstruction of the field while our approach provides only an approximation of the field, this is the reason why we have $M<M_{\mathrm{B}} \ll M_{\mathrm{H}}$. Of course, the proposed approximation approach can be improved by increasing the number $M$ of sampling points.
TABLE I: Number of Spherical Modes and Sampling Points

\begin{tabular}{ccc|cc|ccc}
\hline \hline Ant. & fq. & $a$ & \multicolumn{2}{|c}{ truncation order } & \multicolumn{3}{c}{ nb. of measurements } \\
& {$[\mathrm{GHz}]$} & {$[\mathrm{m}]$} & {$\left[\frac{2 \pi}{\lambda} \cdot a\right]$} & $L$ & $M_{\mathrm{H}}$ & $M_{\mathrm{B}}$ & $M$ \\
\hline RA & 12 & 0.20 & 61 & 55 & 6216 & 2669 & 871 \\
SGH & 26 & 0.04 & 32 & 30 & 1891 & 566 & 339 \\
LL & 12 & 0.26 & 66 & 55 & 12432 & 8746 & 2057 \\
\hline \hline
\end{tabular}

Ant.: antenna, fq.: frequency and nb.: number

Note that the RA and SGH have been characterized over half a sphere.

\section{CONClusion}

A compressive sensing based approach has been applied to speed up the 3D far field characterization of antennas. By exploiting the sparsity of the spherical wave spectrum of antennas, a small number of measurements points are required to interpolate the radiated far field with a reasonable accuracy. Important reduction in terms of measurement point number (up to $70 \%$ compared to standard approaches) are shown for several experimental examples. Finally, sampling strategies enabling fast spherical scans are proposed to render the procedure easy to apply in existing far field measurement facilities.

\section{ACKNOWLEDGMENT}

This publication is supported by the European Union through the European Regional Development Fund (ERDF), and by the french region of Brittany through the CPER Project 
SOPHIE / STIC \& Ondes. The authors would also like to thank Thalès Alenia Space for allowing us to use the 3D far field patterns of their prototypes.

\section{REFERENCES}

[1] D. Donoho, “Compressed sensing," IEEE Trans. on Information Theory, vol. 52, no. 4, pp. 1289-1306, April 2006

[2] E.J. Candès and M.B. Wakin, "An introduction to compressive sampling," IEEE Signal Process. Mag., vol. 25, no. 2, pp. 21-30, Mar. 2008.

[3] http://dsp.rice.edu/cs

[4] A. Massa, P. Rocca, G. Oliveri, "Compressive Sensing in Electromagnetics - A Review," IEEE Antennas and Propagation Mag., vol. 57, no. 1, Feb. 2015.

[5] M.D. Migliore, "A simple introduction to compressed sensing/sparse recovery with applications in antenna measurements," IEEE Antennas and Propagation Magazine, vol. 56, no. 2, pp. 14-26, April 2014.

[6] B. Fuchs, L. Le Coq, and M.D. Migliore, "Fast Antenna Array Diagnosis from a Small Number of Far-Field Measurements," IEEE Trans. Antennas Propag., vol. 64, no. 6, pp. 2227-2235, June 2016.

[7] G. Giordanengo, M. Righero, F. Vipiana, G. Vecchi, M. Sabbadini, "Fast Antenna Testing With Reduced Near Field Sampling," IEEE Trans. Antennas Propag., vol. 62, no. 5, pp. 2501-2513, May 2016.

[8] W.W. Hansen, "A new type of expansion in radiation problems," Phys. Review, Vol. 47, pp. 139-143, January 1935.

[9] J.A. Stratton, "Electromagnetic Theory," New York: McGraw Hill, Chap. 7, 1941.

[10] R. Cornelius, D. Heberling, N. Koep, A. Behboodi, and R. Mathar, "Compressed sensing applied to spherical near-field to far-field transformation," 10th European Conference on Antennas and Propagation (EuCAP), Davos, 2016.

[11] D. Loschenbrand, C. Mecklenbrauker, "Fast Antenna Characterization via a Sparse Spherical Multipole Expansion," 4th International Workshop on Compressed Sensing Theory and its Applications to Radar, Sonar and Remote Sensing (CoSeRa), Aachen, 2016

[12] J.E. Hansen, "Spherical Near-Field Antenna Measurements," Stevenage, U.K.: Peter Peregrinus, 1988.

[13] G.H. Golub and C.F. Van Loan, "Matrix Computations," Baltimore, MD, USA: Johns Hopkins Univ. Press, vol. 3, 2012.

[14] E. van den Berg and M.P. Friedlander, "fSPGL1g: A solver for largescale sparse reconstruction," 2007.

[15] YALL1 basic solver code: Y. Zhang, J. Yang, and W. Yin. YALL1: Your ALgorithms for L1, online at yall1.blogs.rice.edu, 2011.

[16] CVX Research, Inc. CVX: Matlab software for disciplined convex programming, version 2.0 beta. http://cvxr.com/cvx, September 2012.

[17] S. Foucart, H. Rauhut "A Mathematical Introduction to Compressive Sensing," Applied and Numerical Harmonic Analysis, Birkhauser, 2013.

[18] M.D. Migliore, "On the Sampling of the Electromagnetic Field Radiated by Sparse Sources," IEEE Trans. Antennas Propag., vol. 63, no. 2, pp. 553-564, Feb. 2015.

[19] O.M. Bucci, C. Gennarelli, and C. Savarese, "Optimal interpolation of radiated fields over a sphere,” IEEE Trans. Antennas Propag., vol. 39, no. 11, pp. 1633-1643, 1991.

[20] H. Rauhut and R. Ward, "Sparse recovery for spherical harmonic expansions," 9th International Conference on Sampling Theory and Applications (SampTA), 2011.

[21] D. Donoho, J. Tanner, "Precise Undersampling Theorems," Proc. of IEEE, Vol. 98, No. 6, June 2010.

[22] S. Pivnenko, J.E. Pallesen, O. Breinbjerg, M. Sierra-Castaner, P. Caballero, C. Martinez, J.L. Besada, J. Romeu, S. Blanch, J.M. GonzalezArbesu, C. Sabatier, A. Calderone, G. Portier, H. Eriksson, J. Zackrisson, "Comparison of Antenna Measurement Facilities With the DTU-ESA 12 $\mathrm{GHz}$ Validation Standard Antenna Within the EU Antenna Centre of Excellence," IEEE Trans. Antennas Propag., vol. 57, no. 7, pp. 1863 1878, July 2009.

[23] C.D. Diallo, E. Girard, H. Legay, R. Sauleau, and O. Quevedo-Teruel, "All-metal Ku-band Luneburg lens antenna based on variable parallel plate spacing Fakir bed of nails," submitted to European Conference on Antenna and Propagation, 2017. 